\title{
Calculation of optical imbalances in ophthalmic lenses using a new algorithm based on the local dioptric power matrix formalism
}

\author{
José A. Gómez-Pedrero, José Alonso, and Eusebio Bernabeu \\ Department of Optics, Universidad Complutense de Madrid, Facultad de Ciencias Físicas, Ciudad Universitaria s/n, \\ 28040 Madrid, Spain
}

Received August 23, 2000; revised manuscript received November 3, 2000; accepted November 13, 2000

We present a new algorithm to calculate the optical imbalances and differential prismatic effects that appear when two eyes look at an object through correcting eyeglasses. These are important magnitudes in ophthalmic optics because large amounts of them will disturb the binocular vision of the spectacle wearer. As a practical application of our algorithm, the distribution of optical imbalances and differential prismatic powers for a pair of progressive addition lenses has been calculated, and we obtain information about the effects of this kind of lens on the binocular vision of the wearer. (C) 2001 Optical Society of America

OCIS codes: $330.4460,080.2730,330.1400$

\section{INTRODUCTION}

When the eye's ametropia is compensated with an ophthalmic lens, the eye looks at objects through small portions of the lens that could be placed far away from the lens optical center. This is due to the small diameter of the eye's pupil, which in foveal vision limits the size of the light bundles arriving at the retina from the object points. This fact has strong implications in the design of an ophthalmic lens: first, because the off-axis aberrations (mainly oblique astigmatism and field curvature) must be considered locally in order to evaluate their effect in the image quality: second, because the lens surfaces can be designed to provide different sagittal and tangential powers for the different sight directions, which is the case for aspheric and progressive addition lenses.

Besides the effect of the eye rotation on the behavior of the ophthalmic lens as an optical instrument, another important fact that must be taken into account in the design process of a spectacle lens is binocular vision. It is a well-known fact that the human visual system uses the information provided by both eyes to build a single visual sensation. ${ }^{1,2}$ This process, called binocular fusion, is due to the existence of the physiological connection between different zones of the two retinas. The points located in each retina that produce the same visual sensation when they are illuminated by light coming from a single object point are called corresponding points. The binocular fusion process improves considerably the spatial perception ability of the visual system and allows relief perception or stereopsis.

The alterations in the binocular fusion process are directly related to the differences between the two retinal images of an object. ${ }^{1,2}$ These differences may be due to a different amount of ametropia of both eyes (anisometropia) or may be caused by a different perception of the dimensions of the two retinal images (aniseikonia). When ophthalmic lenses are used, it is possible that the shape, size, and position of the two retinal images will be different if the lens power is not the same for the right and the left eye. In this case, we say that there exists an optical imbalance.

It is not only an optical imbalance that may disturb the binocular fusion process. In ophthalmic optics the prismatic effect (or more properly, ray deviation) is defined as the deviation experienced by the central (principal) ray of a narrow light bundle when it passes through a lens. If the prismatic deviation induced by the left-eye and righteye lenses is different enough, a dissociation of the two retinal images of an object will occur and, consequently, binocular fusion will not take place.

As we have said before, when an observer looks at an object through eyeglasses, only small portions of the lenses, which, of course depend on the sight directions, are used. In general, the local power of the left and the right lens at these portions will be different due to the lens aberrations. Thus an optical imbalance will occur, and binocular vision will be altered. It is also possible that the differential effect will be so high that the binocular fusion will be broken up. In summary, to evaluate the performance of a pair of ophthalmic lenses as a binocular optical system, it is necessary to calculate the optical imbalances and differential prismatic powers for a given object point.

Forward or backward ray tracing through an arbitrary single lens is a well-known and straightforward procedure, regardless of whether the computation is exact (numerical) or approximate (numerical or analytical). However, to compute the differential prismatic effect or optical imbalances, we should find the points at which the sight directions of each eye intersects the respective lens surface. This is a complex and time-consuming procedure when exact ray tracing is used.

In this paper we present an algorithm to perform this calculation for any pair of ophthalmic lenses. The algo- 
rithm is based on the local dioptric power matrix formalism, ${ }^{3,4}$ which allows us to calculate analytically the path of a ray through a lens with arbitrary refracting surfaces under certain approximations. ${ }^{3}$ In this way, we can obtain the sight directions of the two eyes when they are looking at a given point through the eyeglasses. This can be done for any kind of ophthalmic lens (spherical, aspherical, toric, and progressive lenses). Once we have determined the sighting directions for the two eyes, it is possible to know the portion of the lens surface that is used to look at the object. Then, using the properties of the local dioptric power formalism, ${ }^{3,4}$ we can compute the local power and the prismatic power at these portions and, consequently, the amount of the optical imbalances. The method can also be used to obtain good seeds as starting points for a precise and time-consuming computation based on exact ray tracing.

As an application example, we have used our algorithm to calculate the distribution of the optical imbalances and differential prismatic powers for a pair of progressive addition lenses when the user is looking at a lattice of object points placed at far, near, and intermediate distances. This allows a numerical evaluation of the binocular performance of progressive addition lenses, and it could be used in the design process of this kind of lens.

Moreover, as the object point (usually an array of object points) can be located anywhere on the object field, we may test the performance of the same pair of lenses when different wearers or observation conditions are considered. Finally, as the algorithm presented is based on the analytical calculation of ray paths through ophthalmic lenses for which refracting surfaces are known, it could be employed to evaluate theoretical designs without making them.

\section{ALGORITHM FOR CALCULATION OF OPTICAL IMBALANCE}

Let us consider the situation depicted in Fig. 1(a). In this figure, $O$ is the object point, $R$ is the rotation center of the right eye, and $L$ is the rotation center of the left eye. According to the reference system of Fig. 1(a), the coordinates of these points are $O,(x, y, z) ; R,(-d, 0,0)$; and $L$, $(d, 0,0)$ with $d$ being the nasopupillary distance, which is assumed to be the same for both eyes. We will consider the principal rays as they have been drawn in Fig. 1(a). These rays pass through the rotation centers of the left and right eyes because we assume that the entrance pupil of the mobile eye is placed at the location of the rotation center, ${ }^{5,6}$ Throughout this discussion we will assume the lenses to be thin, and we call $I^{R}$ and $I^{L}$ the incidence points of the principal rays over the surfaces of the right and left lenses, respectively. As can be seen in Fig. 1(b), the coordinates of $I^{R}$ referring to the geometrical center of the right lens-point $C^{R},\left(-d, 0, l_{2}^{\prime}\right)$-are $I^{R},\left(\xi^{R}, \eta^{R}, 0\right)$ and for the incidence point of the chief ray over the left lens $I^{L},\left(\xi^{L}, \eta^{L}, 0\right)$. The coordinates of these points, referring to the reference system defined in Fig. 1(a), are $I^{R}$, $\left(-d+\xi^{R}, \eta^{R}, l_{2}^{\prime}\right)$ and $I^{L},\left(d+\xi^{L}, \eta^{L}, l_{2}^{\prime}\right)$, with $l_{2}^{\prime}$ being the distance between the vertex of the back lens surface and the rotation center of the eye. The aim of our algorithm is to find the coordinates of the incidence points $I^{R}$ and $I^{L}$, in order to calculate the optical imbalances in the following way. We will assume that the values of spherical and cylindrical power are known for every point at the lens surfaces. This means that we know the functions $E^{R}\left(\xi^{R}, \eta^{R}\right)$ and $C^{R}\left(\xi^{R}, \eta^{R}\right)$, which give the distribution of the spherical and the cylindrical power, respectively, for the right lens. We also know their equivalents for the left lens. Thus optical imbalances may be defined as

$$
\begin{aligned}
& \Delta E(x, y, z)=E^{R}\left(\xi^{R}, \eta^{R}\right)-E^{L}\left(\xi^{L}, \eta^{L}\right), \\
& \Delta C(x, y, z)=C^{R}\left(\xi^{R}, \eta^{R}\right)-C^{L}\left(\xi^{L}, \eta^{L}\right),
\end{aligned}
$$

where $E^{R}\left(\mathbf{r}^{R}\right)$ and $C^{R}\left(\mathbf{r}^{R}\right)$ are the local spherical and the cylindrical power, respectively, of the right lens at the point $I^{R}$ and $E^{L}\left(\mathbf{r}^{L}\right)$, and $C^{L}\left(\mathbf{r}^{L}\right)$ are the corresponding magnitudes for the left lens. In this paper we have used the positive cylinder convention. In a similar way, we have for the differential prismatic deviations

$$
\begin{aligned}
& \Delta P_{x}(x, y, z)=P_{x}^{R}\left(\mathbf{r}^{R}\right)-P_{x}^{L}\left(\mathbf{r}^{R}\right), \\
& \Delta P_{y}(x, y, z)=P_{y}^{R}\left(\mathbf{r}^{L}\right)-P_{y}^{L}\left(\mathbf{r}^{L}\right),
\end{aligned}
$$

where $P_{x}^{R}\left(\mathbf{r}^{R}\right)$ and $P_{x}^{L}\left(\mathbf{r}^{R}\right)$ are the horizontal components of the prismatic deviation for the right- and left-eye lenses and $P_{y}^{R}\left(\mathbf{r}^{L}\right)$ and $P_{y}^{L}\left(\mathbf{r}^{L}\right)$ are the vertical ones. Note that

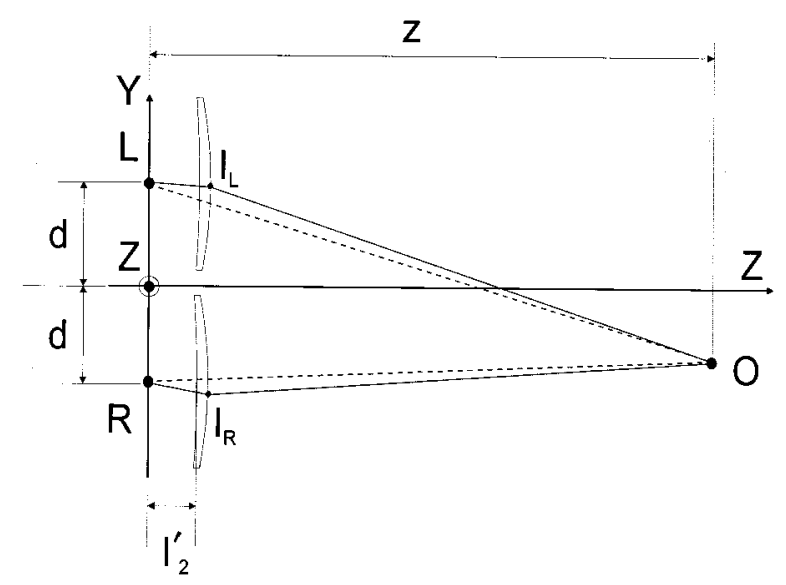

(a)

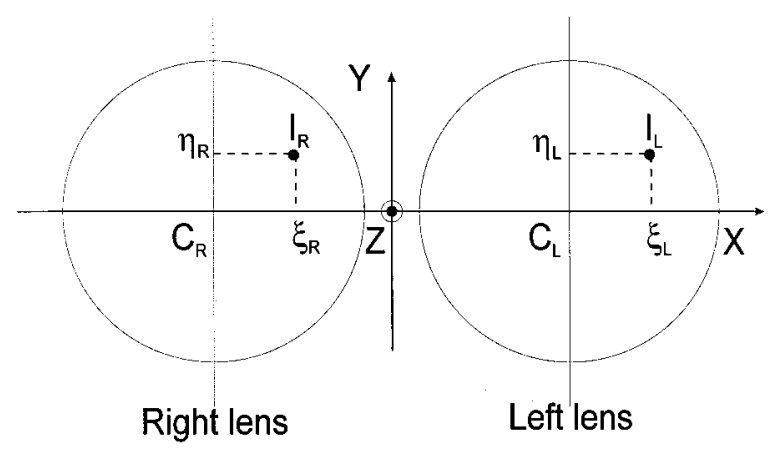

(b)

Fig. 1. (a) Scheme of the principal rays' path from the object point $\mathrm{O}$ to the left- and right-eye rotation centers: $d$ is the nasopupillary distance (assumed equal for the two eyes), $z$ is the object distance, and $l_{2}^{\prime}$ is the distance between the back surface of the lens and the rotation center of the eye. (b) Plot representing the coordinate system employed, referring to the respective centers of left and right lenses. 
the two optical imbalances $\Delta E, \Delta C$ and the differential prismatic powers $\Delta P_{x}, \Delta P_{y}$ are functions of the coordinates of the object point $O$. We can use this functional dependence to establish the extension of the object field of the lens/eye system from a physiological point of view; that is, we will define the object field as the set of object points for which the optical imbalances and differential prismatic powers have numerical values that can be tolerated by the average human visual system.

To calculate the coordinates of $I^{R}$ and $I^{L}$ we have to consider the ray vector for the incident and the refracted principal ray for both the right and the left lens. For the right lens, the coordinates of these vectors are

$$
\begin{aligned}
\mathbf{k i}^{R} & =\frac{\left(d+\xi^{R}-x, \eta^{R}-y, l_{2}^{\prime}-z\right)}{\left[\left(d+\xi^{R}-x\right)^{2}+\left(\eta^{R}-y\right)^{2}+\left(l_{2}^{\prime}-z\right)^{2}\right]^{1 / 2}}, \\
\mathbf{k r}^{R} & =\frac{\left(-\xi^{R},-\eta^{R}, l_{2}^{\prime}\right)}{\left[\left(\xi^{R}\right)^{2}+\left(\eta^{R}\right)^{2}+\left(l_{2}^{\prime}\right)^{2}\right]^{1 / 2}} .
\end{aligned}
$$

Assuming that $\left(l_{2}^{\prime}-z\right)^{2} \gg\left(d+\xi^{R}-x\right)^{2}+\left(\eta^{R}-y\right)^{2}$ and $\left(l_{2}^{\prime}\right)^{2} \gg\left(\xi^{R}\right)^{2}+\left(\eta^{R}\right)^{2}$, we can write Eqs. (3a) and (3b) as

$$
\begin{aligned}
\mathbf{k i}^{R} & =\left(\frac{d+\xi^{R}-x}{z-l_{2}^{\prime}}, \frac{\eta^{R}-y}{z-l_{2}^{\prime}},-1\right), \\
\mathbf{k r}^{R} & =\left(\frac{-\xi^{R}}{l_{2}^{\prime}}, \frac{-\eta^{R}}{l_{2}^{\prime}},-1\right) .
\end{aligned}
$$

By definition, the horizontal and vertical prismatic powers at the point $I_{R}$ are given by

$$
\begin{aligned}
& P_{x}\left(\mathbf{r}^{R}\right)=k r_{x}^{R}-k i_{x}^{R}, \\
& P_{y}\left(\mathbf{r}^{R}\right)=k r_{y}^{R}-k i_{y}^{R} .
\end{aligned}
$$

After substituting the components of the incident and refracted ray vectors as given by Eqs. (4a) and (4b), we arrive, after straightforward calculations, to the following equation system:

$$
\begin{aligned}
& P_{x}\left(\mathbf{r}^{R}\right)+\left(\frac{1}{z-l_{2}^{\prime}}+\frac{1}{l_{2}^{\prime}}\right) \xi^{R}-\frac{x-d}{z-l_{2}^{\prime}}=0, \\
& P_{y}\left(\mathbf{r}^{R}\right)+\left(\frac{1}{z-l_{2}^{\prime}}+\frac{1}{l_{2}^{\prime}}\right) \eta^{R}-\frac{y}{z-l_{2}^{\prime}}=0 .
\end{aligned}
$$

For the left lens, we have a similar, but independent, equation system:

$$
\begin{aligned}
& P_{x}\left(\mathbf{r}^{L}\right)+\left(\frac{1}{z-l_{2}^{\prime}}+\frac{1}{l_{2}^{\prime}}\right) \xi_{L}-\frac{x+d}{z-l_{2}^{\prime}}=0, \\
& P_{y}\left(\mathbf{r}^{L}\right)+\left(\frac{1}{z-l_{2}^{\prime}}+\frac{1}{l_{2}^{\prime}}\right) \eta_{L}-\frac{y}{z-l_{2}^{\prime}}=0 .
\end{aligned}
$$

From these equation systems we obtain the position vectors $\mathbf{r}^{R}=\left(\xi^{R}, \eta^{R}\right)$ and $\mathbf{r}^{L}=\left(\xi_{L}, \eta_{L}\right)$ of the points $I_{R}$ and $I_{L}$, and we calculate the optical imbalances and prismatic power differences using Eqs. (1) and (2).

We will denote the preceding equation systems in a shortened form as

$$
\begin{aligned}
& \mathbf{S}^{R}\left(\mathbf{r}^{R}\right)=\mathbf{0}, \\
& \mathbf{S}^{L}\left(\mathbf{r}^{L}\right)=\mathbf{0},
\end{aligned}
$$

with $\mathbf{S}^{R}\left(\mathbf{r}^{R}\right)$ and $\mathbf{S}^{L}\left(\mathbf{r}^{L}\right)$ being two vector functions whose explicit expressions are

$$
\begin{aligned}
& \mathbf{S}^{R}\left(\mathbf{r}^{R}\right)=\left[\begin{array}{c}
P_{x}\left(\mathbf{r}^{R}\right)+\left(\frac{1}{z-l_{2}^{\prime}}+\frac{1}{l_{2}^{\prime}}\right) \xi^{R}-\frac{x-d}{z-l_{2}^{\prime}} \\
P_{y}\left(\mathbf{r}^{R}\right)+\left(\frac{1}{z-l_{2}^{\prime}}+\frac{1}{l_{2}^{\prime}}\right) \eta^{R}-\frac{y}{z-l_{2}^{\prime}}
\end{array}\right], \\
& \mathbf{S}^{L}\left(\mathbf{r}^{L}\right)=\left[\begin{array}{c}
P_{x}\left(\mathbf{r}^{L}\right)+\left(\frac{1}{z-l_{2}^{\prime}}+\frac{1}{l_{2}^{\prime}}\right) \xi^{L}-\frac{x-d}{z-l_{2}^{\prime}} \\
P_{y}\left(\mathbf{r}^{L}\right)+\left(\frac{1}{z-l_{2}^{\prime}}+\frac{1}{l_{2}^{\prime}}\right) \eta^{L}-\frac{y}{z-l_{2}^{\prime}}
\end{array}\right] .
\end{aligned}
$$

Although there are explicit (and approximate) expressions for the prismatic power components of spherical, aspherical, and spherocylindrical lenses, ${ }^{7}$ these expressions are too complicated to find an analytical solution of the equation systems (8a) and (8b). Moreover, in the case of progressive addition lenses we do not have, in general, an analytical expression for the prismatic power components. This implies that we have to solve the equation systems numerically. To do this, we employed the algorithm described in Press et al., 8 which is a NewtonRaphson method for nonlinear equation system. This method makes use of the Jacobian of the function $\mathbf{S}^{R}\left(\mathbf{r}^{R}\right)$, which is defined as

$$
\mathbf{J}^{R}\left(\mathbf{r}^{R}\right)=\left[\begin{array}{ll}
\partial_{\xi} S_{x}\left(\mathbf{r}^{R}\right) & \partial_{\eta} S_{x}\left(\mathbf{r}^{R}\right) \\
\partial_{\xi} S_{y}\left(\mathbf{r}^{R}\right) & \partial_{\eta} S_{y}\left(\mathbf{r}^{R}\right)
\end{array}\right],
$$

where $S_{x}\left(\mathbf{r}^{R}\right)$ and $S_{y}\left(\mathbf{r}^{R}\right)$ are the components of function $\mathbf{S}^{R}\left(\mathbf{r}^{R}\right)$. Substituting the expressions for $S_{x}\left(\mathbf{r}^{R}\right)$ and $S_{y}\left(\mathbf{r}^{R}\right)$ given on the left side of Eq. (9a), in Eq. (10) we find that

$$
\mathbf{J}^{R}\left(\mathbf{r}^{R}\right)=\left[\begin{array}{ll}
\partial_{\xi} P_{x}\left(\mathbf{r}^{R}\right) & \partial_{\eta} P_{x}\left(\mathbf{r}^{R}\right) \\
\partial_{\xi} P_{y}\left(\mathbf{r}^{R}\right) & \partial_{\eta} P_{y}\left(\mathbf{r}^{R}\right)
\end{array}\right]+\left(\frac{1}{z-l_{2}^{\prime}}+\frac{1}{l_{2}^{\prime}}\right) \mathbf{I},
$$

with $\mathbf{I}$ being the $2 \times 2$ identity matrix. Finally, we can write Eq. (11) as

$$
\mathbf{J}^{R}\left(\mathbf{r}^{R}\right)=\mathbf{F}^{R}\left(\mathbf{r}^{R}\right)+\left(\frac{1}{z-l_{2}^{\prime}}+\frac{1}{l_{2}^{\prime}}\right) \mathbf{I},
$$

where $\mathbf{F}^{R}\left(\mathbf{r}^{R}\right)$ is the local dioptric power matrix of the right lens. In the derivation of Eq. (12) we used the properties of the local dioptric power matrix. ${ }^{3,9}$ There is, of course, a similar equation for the Jacobian of the left-eye function $\mathbf{S}^{L}\left(\mathbf{r}^{L}\right)$. Since there is a simple relationship between the local dioptric power matrix and the prismatic power components, 3,9 Eq. (12) allows for an easier and faster calculation of the Jacobian and, consequently, of the equation system solution. As the Newton-Raphson 
algorithm states, to solve equation systems like Eqs. (8a) and $(8 \mathrm{~b})$, we have to follow these steps:

1. First we must state a seed solution; let us call it $\mathbf{r}_{0}^{R}$ (or $\mathbf{r}_{0}^{L}$ for the left-eye equation system). In our case, we select $\mathbf{r}_{0}^{R}$ as the position vector of the point at which the straight line that joins the object point and the rotation center of the eye intersects the spectacle plane.

2. From the seed solution, we calculate a new solution as $\mathbf{r}_{1}^{R}=\mathbf{r}_{0}^{R}+\delta \mathbf{r}$, where $\delta \mathbf{r}=-\left[\mathbf{J}^{R}\left(\mathbf{r}_{0}^{R}\right)\right]^{-1} \cdot \mathbf{S}^{R}\left(\mathbf{r}_{0}^{R}\right)$. (This is why we have to compute the Jacobian matrix.)

3. The last step is to calculate $\mathbf{S}^{R}\left(\mathbf{r}_{1}^{R}\right)$; if the value found is not zero (below certain tolerance level, of course), we repeat steps 1 and 2 (with $\mathbf{r}_{1}^{R}$ as the new seed solution) until the algorithm converges.

To summarize, the use of the local dioptric power matrix formalism permits the calculation of the optical imbalances and differential prismatic powers for any pair of ophthalmic lenses at any location of the object point.

\section{APPLICATION TO PROGRESSIVE ADDITION LENSES}

It is well known that progressive addition lenses (PAL's) present a continuous variation of the local spherical power. This power variation is distributed in a very specific way to compensate the presbyopia of the lens user. At the same time, the lens power variation should respect the convergence-accommodation relationship of the visual system. This is why a PAL presents a zone of approximately quasi-constant power (which compensates the ametropia of the user for far viewing distances) in the upper half of the lens, a zone of constant power (which compensates the ametropia of the user for near viewing distances) located at the low nasal portion of the lens, and a progressive corridor between these zones.

Besides the distribution of local spherical power, the PAL presents a distribution of local cylindrical power as a result of the nature of the refracting surfaces of this kind of lens. This local cylindrical power is distributed in such a way that its maximum values are reached at both sides of the progressive corridor. In this way, when a PAL user looks at objects placed at lateral zones of the visual field, he or she will experience a loss of visual acuity due to the local cylindrical power. In general, this loss of visual acuity is well tolerated by the human visual system if the optical imbalances and the differential prismatic powers have low numerical values. ${ }^{10}$ Thus the main task of a PAL designer is to provide distributions of local spherical and cylindrical powers with reduced values of the optical imbalances $\Delta E, \Delta C$, and differential prismatic powers $\Delta P_{x}, \Delta P_{y}$.

In this context, we find an application of our algorithm, because it is intended to calculate the optical imbalances and the differential prismatic power of a pair of lenses independent of the type of spectacle lens considered. Therefore we computed the distribution of optical imbalances and differential prismatic powers presented by a pair of commercial PAL lenses (BBGR Selective) as functions of the regarding directions. The lenses employed were acquired directly from the manufacturer. To perform these calculations, we measured the surfaces of the
PAL pair with an automatic topographic system developed by us, ${ }^{4}$ and we obtained from these measurements the distributions of local cylindrical and spherical powers and the components of the prismatic power for both the right and the left lens using the properties of the local dioptric power matrix formalism. ${ }^{3}$ Specifically, we employed an automatic profilometer to measure the shape of the refracting surfaces of the lenses. From these topographic measurements, we obtain a set of discrete values of functions $z_{1}(x, y)$ and $z_{2}(x, y)$, which give the sagittas of the front and back surfaces of the lens. Once we obtained the sagitta values, we calculated from them the first- and second-order derivatives of function ${ }^{3} \delta z=(n$ $-1)\left(z_{1}-z_{2}\right)$, where $n$ is the lens refractive index. These derivatives are related $^{3}$ to local prismatic effects (first-order derivatives of $\delta z$ ) and to local spherical and cylindrical powers (second-order derivatives of $\delta z$ ). In summary, we obtained the distributions (within a discrete set of surface points) of the local refractive and prismatic powers for the pair of lenses considered. The results of the technique employed are equivalent to those given by optical methods such as moiré deflectometry and authomatic focimeter measurements. In a previous study we measured one of the lenses, specifically the right one, with an automatic focimeter, and we presented ${ }^{3}$ the conventional graphics showing the distributions of spherical, cylindrical powers and modulus of prismatic power found. As we have said above, our topographic technique gives equivalent results. Once the local powers have been measured, we can make use of our algorithm to calculate optical imbalances and differential prismatic powers for a given object point location.

We have used a square array of equally spaced object points placed in a plane perpendicular to the $O Z$ direction. We have used the Luneburg's coordinate system ${ }^{11}$ in order to state the position of a single object point. Thus the coordinates of an object point are $\left(\omega_{x}, \omega_{y}, z\right)$, with $\omega_{x}$ and $\omega_{y}$ being the horizontal and vertical angles that specify the sighting direction and $z$ being the viewing distance. In this way, we can relate easily the optical impairments to the lateral displacements of the object point with respect to the spectacle wearer's head. To evaluate the performance of the PAL pair we have chosen three square nets, located at different distances $(z=200 \mathrm{~cm}$, far viewing distance; $z=60 \mathrm{~cm}$, intermediate distance; and $z=37.5 \mathrm{~cm}$, a near viewing distance).

The spatial locations (given by the extreme values of the viewing angles $\omega_{x}$ and $\omega_{y}$ ) of the three arrays were different. For points placed at the far viewing distance, the values of $\omega_{y}$ oscillated between 0 and $25^{\circ}$. For intermediate points, the extreme values for $\omega_{y}$ were 0 and $-17^{\circ}$, and for near points, the vertical angle varied between -24 and $-35^{\circ}$. In this way, we have taken into account the convergence-accommodation relationship for the pair of PAL's, and we have assured the correct correspondence between viewing distances and the PAL viewing zones. As an example of the latter assertion, for the points placed at the far viewing distance the chosen values for the angle $\omega_{y}$ ensure that the viewing directions intercept the lens surfaces at points placed in the upper half of the lens, which is the PAL lens zone designed specifically to look at points placed far away from the observer. 
The extreme values of $\omega_{x}$ are $-20^{\circ}$ and $20^{\circ}$ for all viewing distances, which gives a horizontal field of view of $40^{\circ}$.

Once we established the object point locations, we used our algorithm to calculate the coordinates of the points $I^{R}$ and $I^{L}$ for every object point on a given array. In our calculations we supposed that the PAL pair was used by a subject with a nasopupillary distance $d=32 \mathrm{~mm}$ and a distance between back lens surface and center of rotation of the eye of $l_{2}^{\prime}=27 \mathrm{~mm}$. Given the coordinates of the points $I^{R}$ and $I^{L}$, we calculated the values of the optical imbalances and differential prismatic deviations. The results are presented in Figs. 2-4. In these figures we have plotted horizontal profiles of the physiological magnitudes studied for a discrete set of values of the vertical viewing angle $\omega_{y}$. Our aim is to represent the variation of the optical imbalances and differential prismatic power with horizontal eye movements (angle $\omega_{x}$ ) for fixed values of $\omega_{y}$. This is the typical behavior found in common tasks such as reading and writing.

For all three physiological magnitudes studied, we have found a similar pattern [see Figs. 2(a)-2(c)]. The values of both the optical imbalances and the differential prismatic power are near zero for frontal vision (that is, for $\omega_{x}=0^{\circ}$ ), and they rise when the user looks laterally. In other words, the graphics indicate the values of the horizontal viewing angle, for which binocular vision is not significantly impaired by a differential spherical (cylindrical) power or by a differential prismatic effect. In this way we can establish the extent of the horizontal visual fields by following a physiological criterion. We can also study the variation of these visual fields with the vertical viewing angle and compare the behavior of the different (near, far, and intermediate) zones of the PAL examined.

We first studied the behavior of the differential spherical power $\Delta E$. In order to compute the effect of a certain amount of differential spherical power, we must make use of a classical equation ${ }^{6}$ of ophthalmic optics that relates the magnification of an ophthalmic lens to the lens power

$$
m=1-d_{v} E,
$$

with $E$ being the lens spherical power and $d_{v}$ being the distance between the entrance pupil of the eye and the vertex of the back surface of the lens (this magnitude is often assumed to be $d_{v}=-1.5^{-2} \mathrm{~m}$ ). If the powers of the right and left lenses are different, the lenses' magnifications will be different, and consequently the retinal images will have different sizes (aniseikonia). If there exists a difference of more than $5 \%$ between the magnification of the left and right lenses, binocular vision will be altered. ${ }^{1}$ From Eq. (13) we can write the relative differential magnification as

$$
\delta m=\frac{m_{R}-m_{L}}{m_{R}}=\frac{d_{v}|\Delta E|}{1-d_{v} E_{R},}
$$

with $E_{R}$ being the power of the right lens and $|\Delta E|$ being the absolute value of the differential spherical power. As shown in Fig. 2b, we find peak values of $\Delta E$ of $\sim 0.5$ diopters (D), with a lens power in this zone (intermediate portion of the PAL) of approximately $+1 \mathrm{D}$. Substituting into Eq. (14) we find a relative differential magnification $\delta m=7 \times 10^{-3}$, that is, a relative magnification differ- ence of $0.7 \%$. This is lower than the $5 \%$ of magnification difference tolerated by the human visual system. So we can assume that no appreciable impairments due to aniseikonia will be found.

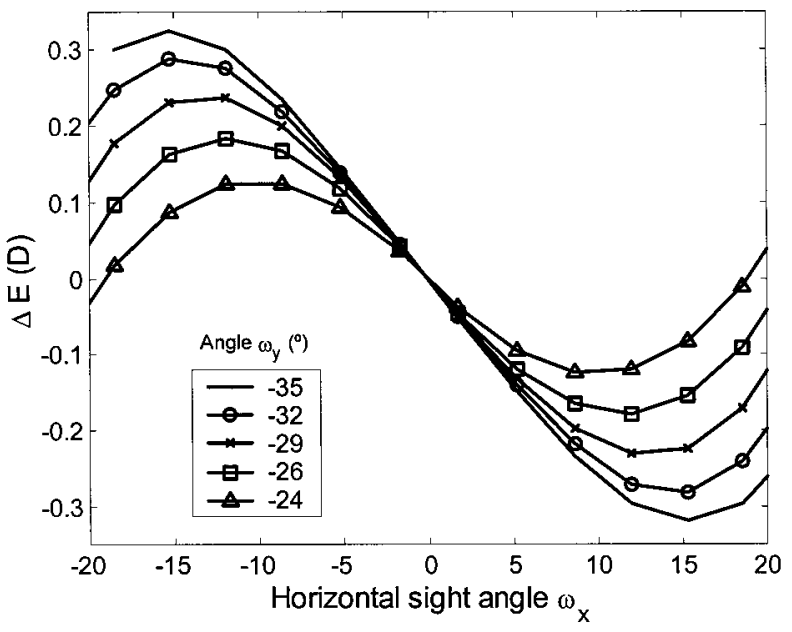

(a)

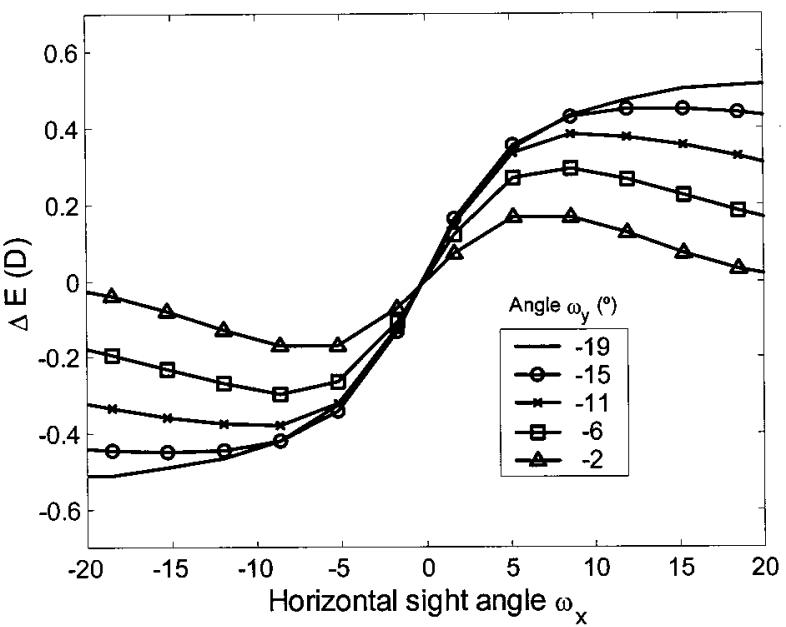

(b)

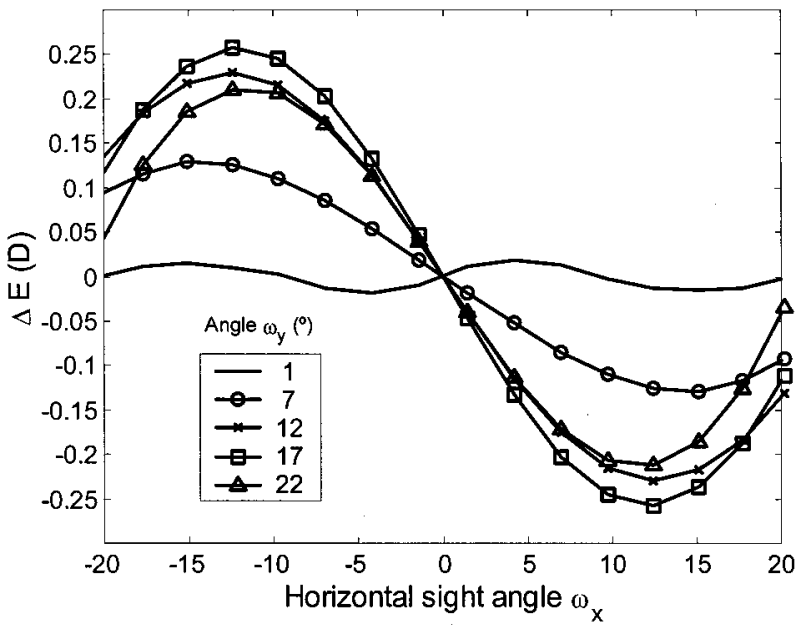

(c)

Fig. 2. Plots of the calculated differential spherical power versus the horizontal sight angle $\omega_{x}$ for three object distances: (a) near, (b) intermediate, and (c) far. Each line indicates a different value of the vertical sight angle. 


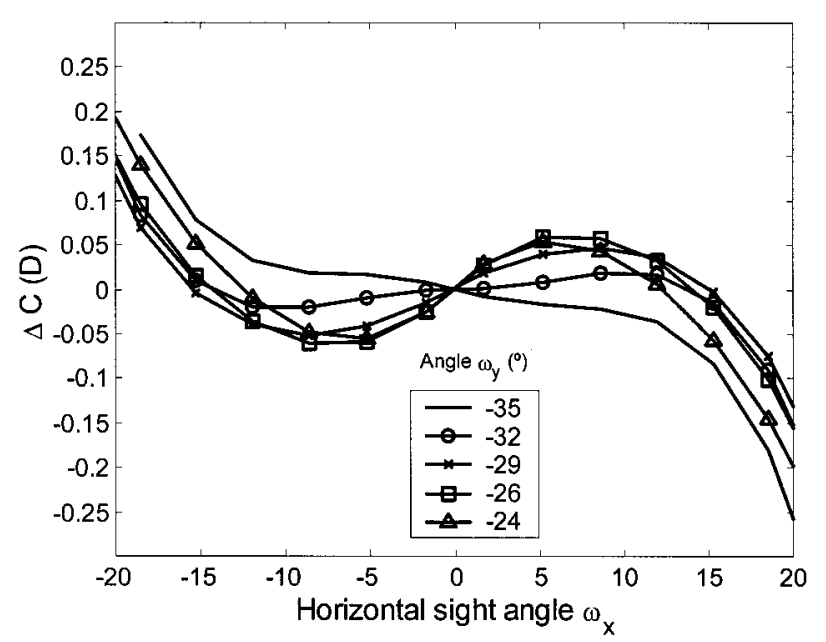

(a)

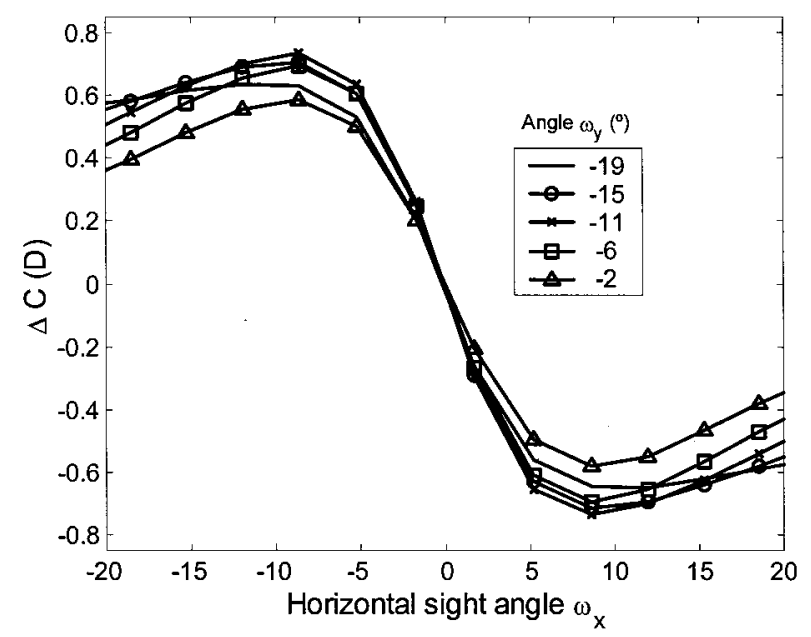

(b)

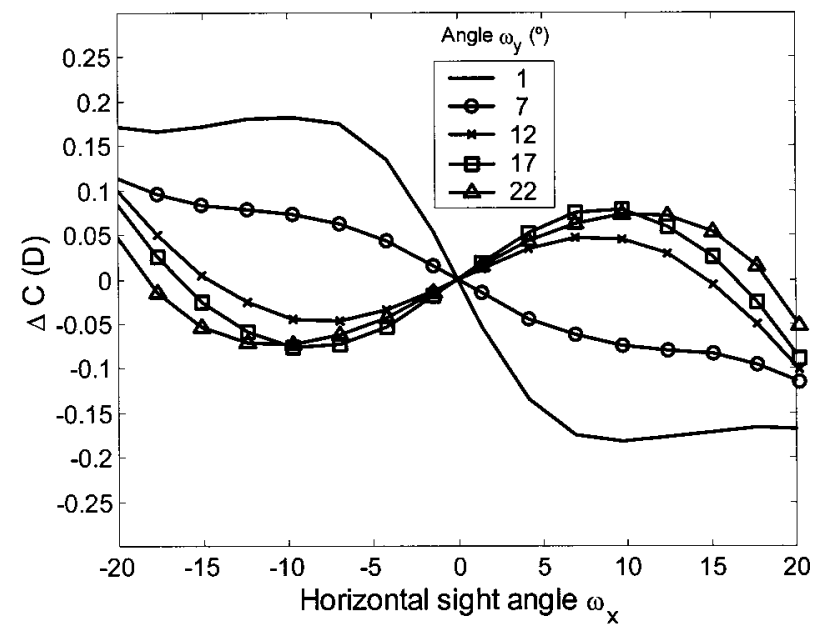

(c)

Fig. 3. Plots of the calculated differential cylindrical power versus the horizontal sight angle $\omega_{x}$ for three object distances: (a) near, (b) intermediate, and (c) far. Each line indicates a different value of the vertical sight angle.

With the exception of the work of Rassow and Weseman, ${ }^{10}$ we have not found in the literature any relationship between a certain amount of cylindrical imbal-

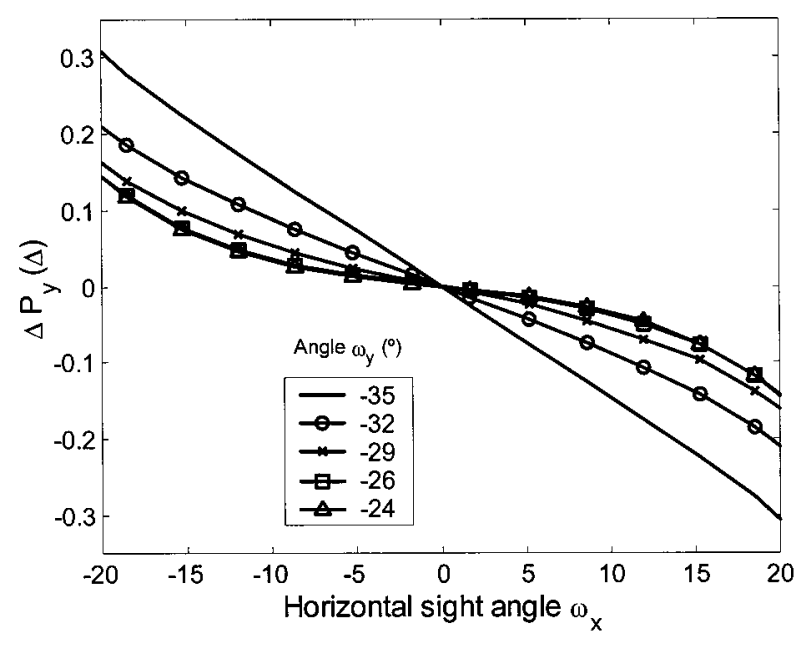

(a)

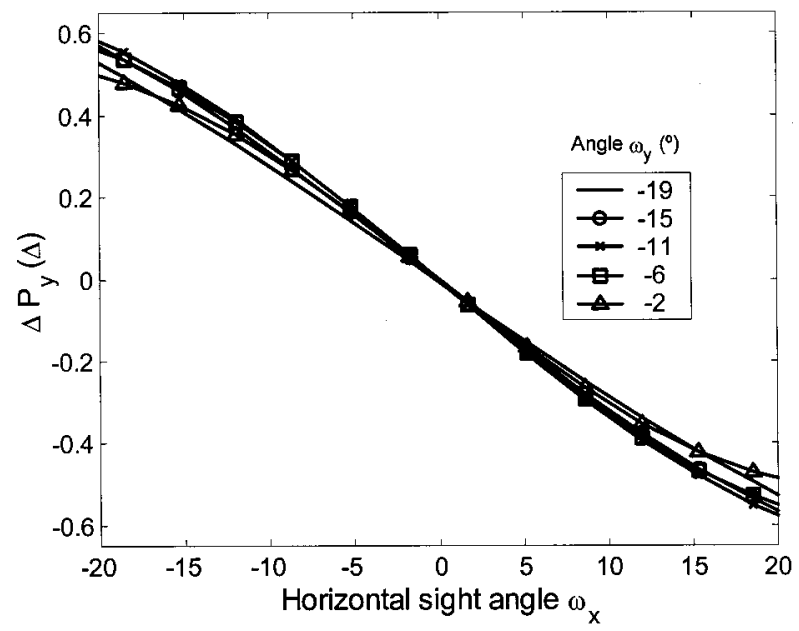

(b)

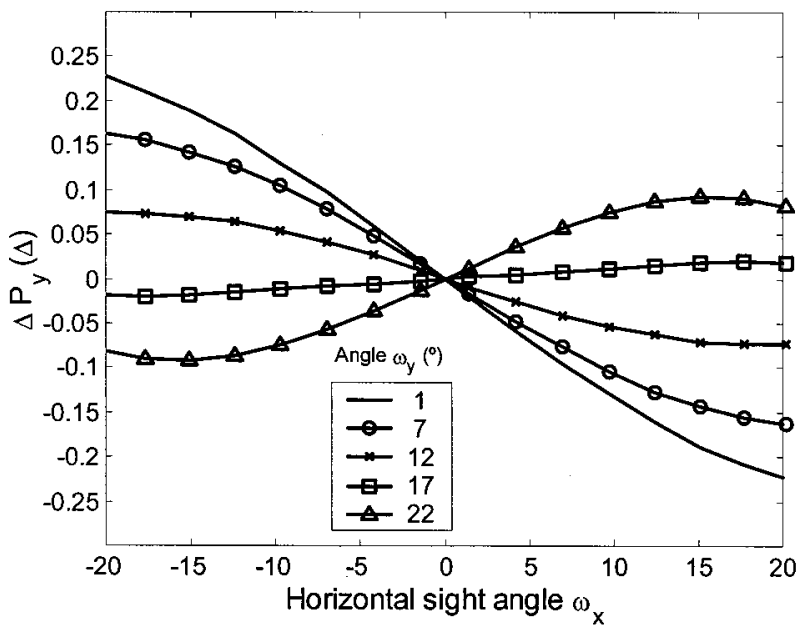

(c)

Fig. 4. Plots of the calculated vertical differential prismatic power versus the horizontal sight angle $\omega_{x}$ for three object distances: (a) near, (b) intermediate, and (c) far. Each line indicates a different value of the vertical sight angle.

ance and the consequent loss of binocular abilities of the visual system. In the cited work a cylindrical imbalance of $\pm 0.5 \mathrm{D}$ raises the depth perception threshold by 20 arc 
sec. In terms of impairment of the binocular system, this effect is equivalent to $6 \%$ relative differential magnification between the right and left retinal images. Thus we could set this value of $\pm 0.5 \mathrm{D}$ as the threshold of cylindrical imbalance, because this amount of cylindrical imbalance severely impairs the binocular vision of the spectacle wearer. According to the differential cylindrical power distribution shown in Figs. 3(a)-3(c), we will find a considerable limitation in the extent of the horizontal visual fields for the points placed at intermediate viewing distances. From the plots of Fig. 3(b), given the tolerance threshold of $\pm 0.5 \mathrm{D}$, we found a horizontal visual field extension of $10^{\circ}$ (from $-5^{\circ}$ to $+5^{\circ}$ ) for all the values of $\omega_{y}$ corresponding to the intermediate portion of the PAL. To compare, we do not find a differential cylindrical power higher than $\pm 0.3 \mathrm{D}$ for the whole range of values of $\omega_{x}$ (from $-20^{\circ}$ to $+20^{\circ}$ ) in both near and far zones of the lens [Figs. 3(a) and 3(c), respectively].

Finally, we present in Fig. 4 the distribution of the vertical differential prismatic power, $\Delta P_{y}$ as function of the viewing angle $\omega_{x}$. Once again, maximum values of $\Delta P_{y}$ are found in the intermediate zone for extreme values of $\omega_{x}$. In any case, the numerical values of $\Delta P_{y}$ never reach the threshold value of $1 \Delta$, which has been extensively reported in the literature ${ }^{6,12}$ as the tolerance value for vertical differential prismatic power. ${ }^{13}$ Thus we can conclude that according to the calculated distribution of $\Delta P_{y}$, the PAL will not introduce great distortions of binocular vision, as a result of the differential prismatic powers for the whole extension of the visual fields for which these distributions have been calculated.

\section{CONCLUSIONS}

We have developed a new algorithm to calculate the optical imbalances and differential prismatic power experienced by the human vision system when looking at an object through a pair of eyeglasses. The computation of these physiological magnitudes is important in ophthalmic optics because they have a great influence in the spectacle-lenses wearer's binocular vision. Moreover, our algorithm is intended to be used with any kind of ophthalmic lens, independent of the type of spectacle lens considered. Thus we can calculate the optical imbalances and differential prismatic powers for spherical, spherotorical, aspherical, and, indeed, progressive addition lenses. We must point out that our algorithm has been developed within the frame of the thin-lens approximation. This implies that we will obtain accurate results only for a limited range of lens power. In other words, we could not apply our algorithm to high-powered lenses. However, our algorithm gives accurate results for a range of powers great enough to include the lenses commonly employed in ophthalmic practice.

The algorithm developed presents some advantages over an algorithm based in the exact computation of the ray paths. First, if we want to find the path of the principal ray with an algorithm based on the exact computation of the ray paths, we must employ a recurrence procedure. This implies that we have to trace an undetermined number of rays through the lens in order to find the ray that when departing from the object point passes through the rotation center of the eye. With our algorithm we have to solve only two simple equation systems given by Eqs. (8a) and (8b). Moreover, in some simple cases (lenses with paraboloidal surfaces), it is possible to find analytically the principal ray paths from our algorithm.

Finally, we have computed the optical imbalances and differential prismatic power for a pair of commercial progressive lenses for the viewing directions corresponding to a set of object points placed in a square net at three different viewing distances. In this way, we can evaluate the binocular vision performance of this pair of PAL's attending to the design characteristics of this kind of ophthalmic lens, that are intended to provide the user with a clear vision for a wide range of object distances.

The distributions of optical imbalances and differential prismatic powers for a range of viewing distances plotted in Figs. 3 and 4, give us some interesting indications about the binocular vision behavior of a pair of commercial PAL's. We have found zero values for the optical imbalances and the vertical component of the differential prismatic power for objects placed at the middle of the PAL user's vision field. Thus the PAL user looking at frontal objects does not experience distortions in his binocular vision. These binocular vision distortions appear when the user looks at objects placed laterally with respect to the main sight line. Then the horizontal extension of the visual fields are limited by high values of the optical imbalances or vertical differential prismatic powers. In contrast, visual fields are not limited in vertical direction by these binocular magnitudes, because they do not change appreciably with vertical eye movements.

\section{ACKNOWLEDGMENTS}

We thank the Ministerio de Educación y Ciencia (Spain), for the financial support of this work within the project TAP98-0701.

Corresponding author J. A. Gómez-Pedrero can be reached at Universidad Complutense de Madrid, Escuela de Optica, Avenida Arcos de Jalón s/n, 28037 Madrid, Spain; by telephone, 34-1-394-4403; by fax, 34-1-3944683 ; or by e-mail, fiopt18@sis.ucm.es.

\section{REFERENCES}

1. Y. LeGrand, Optique Physiologique, la dioptrique de l'oeil et sa correction, $3^{\text {ème }}$ ed. (Masson, Paris, 1965).

2. R. W. Reading, Binocular Vision: Foundations and Applications (Butterworth, Boston, Mass, 1983).

3. J. A. Gómez-Pedrero, J. Alonso, H. Canabal, and E. Bernabeu, "A generalization of Prentice's law for ophthalmic lenses with arbitrary refracting surfaces," Ophthalmic Physiol. Opt. 18, 514-520 (1998).

4. J. A. Gómez-Pedrero, PhD. dissertation (Universidad Complutense de Madrid, Madrid, Spain 1999).

5. T. Fannin and T. Grosvernor, Clinical Optics, 1st ed. (Butterworth-Heinemann, Boston, Mass., 1996).

6. M. Jalie, The Principles of Ophthalmic Lenses, 4th ed. (ABDO, London, 1988).

7. J. A. Gómez-Pedrero, J. Alonso, and E. Bernabeu, "Local dioptric power matrix and prismatic effects of spherical, aspherical, and spherotorical ophthalmic lenses," in OPTIKA 
98: 5th Conference on Modern Optics, G. Akos, G. Lupkovics, and A. Podmaniczky, eds. Proc. SPIE 3573, 405-408 (1998).

8. W. H. Press, S. A. Teukolsky, W. Vettering, and B. P. Flannery, Numerical Recipes in C, 2nd ed. (Cambridge U. Press, Cambridge, UK, 1992).

9. J. Alonso, J. A. Gómez-Pedrero, and E. Bernabeu, "Local dioptric power matrix in progressive addition lenses," Ophthalmic Physiol. Opt. 17, 522-529 (1998).
10. B. Rassow and W. Wesemann, "Fusion and stereopsis under artificially impaired conditions of the binocular system," Optometrie 2, 21-28 (1989).

11. R. K. Luneburg, "The metric of binocular visual space," J. Opt. Soc. Am. 40, 627-642 (1950).

12. K. N. Ogle, "Stereopsis and vertical disparity," A. M. A. Arch. Opthal. 53, 495-504 (1955).

13. K. N. Ogle, "Distortion of the image by prism," J. Opt. Soc. Am. 41, 1023-28 (1951). 